\title{
D. Higham, P. Kloeden: "An Introduction to the Numerical Simulation of Stochastic Differential Equations"
}

\section{SIAM, 2021, xvi+277 pp.}

\section{Andreas Neuenkirch ${ }^{1}$}

Accepted: 28 September 2021 / Published online: 11 October 2021

(c) The Author(s) 2021

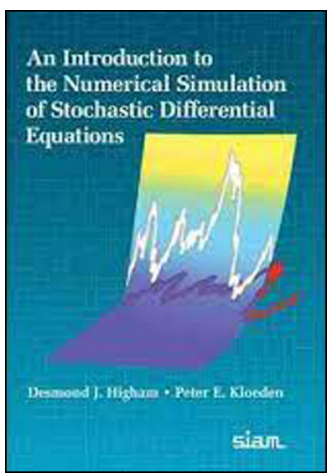

The book under review is an introduction to the numerical simulation of stochastic differential equations (SDEs). In recent years SDEs have become important modeling tools in various application areas as, e.g., engineering, finance, physics, chemistry, biology and social sciences. Loosely speaking, SDEs extend the classical deterministic ordinary differential equations (ODEs) by adding a noise term. More precisely, in the one-dimensional case an SDE reads as

$$
\begin{aligned}
& d X(t)=f(X(t)) d t+g(X(t)) d W(t), \quad t \in[0, T], \\
& X(0)=x_{0} \in \mathbb{R},
\end{aligned}
$$

where $f, g: \mathbb{R} \rightarrow \mathbb{R}$ and $W=(W(t))_{t \in[0, T]}$ is a Brownian motion. The latter is a stochastic process, which satisfies the following properties:

(i) $W$ is a random variable with values in $C([0, T] ; \mathbb{R})$ and satisfies $W(0)=0$, i.e., each path of $W$ is a real-valued continuous function on $[0, T]$ which has the value zero at $t=0$;

(ii) for all $n \in \mathbb{N}$ and $0 \leq t_{0}<t_{1}<t_{2}<\cdots<t_{n} \leq T$ the law of the random variable

$$
\left(W\left(t_{1}\right)-W\left(t_{0}\right), W\left(t_{2}\right)-W\left(t_{1}\right), \ldots, W\left(t_{n}\right)-W\left(t_{n-1}\right)\right)
$$

$\triangle$ A. Neuenkirch

neuenkirch@math.uni-mannheim.de

1 Mannheim, Germany 
is Gaussian with mean $\mu=0 \in \mathbb{R}^{n}$ and covariance matrix

$$
\Sigma=\left(\begin{array}{cccc}
t_{1}-t_{0} & 0 & \cdots & 0 \\
0 & t_{2}-t_{1} & \ddots & \vdots \\
\vdots & \ddots & \ddots & 0 \\
0 & \cdots & 0 & t_{n}-t_{n-1}
\end{array}\right) \in \mathbb{R}^{n, n},
$$

i.e., non-overlapping increments of Brownian motion are independent scalar Gaussian random variables with mean zero and variance given by the length of the time interval.

The name Brownian motion goes back to the Scottish botanist Robert Brown, who in 1827 observed the erratic behavior of pollen grains in water. The development of a mathematical theory for Brownian motion involves contributors as Albert Einstein, Norbert Wiener and Paul Lévy.

The functions $f$ and $g$ in equation (1) are called drift and diffusion coefficient, respectively. As mentioned above, for $g=0$ one recovers an ODE. But what is the mathematical meaning of equation (1)? This question was addressed by the Japanese mathematician Itō Kiyoshi in the 1940s [3, 4] by transferring equation (1) into the integral equation

$$
X(t)=x_{0}+\int_{0}^{t} f(X(s)) d s+\int_{0}^{t} g(X(s)) d W(s), \quad t \in[0, T],
$$

and by developing a theory of stochastic integration to make sense of the object

$$
\text { “ } \int_{0}^{t} g(X(s)) d W(s) " .
$$

In honor of his fundamental contributions, which pioneered the mathematical field of stochastic analysis, this integration theory is now called Itō integration and equation (1) is called an Itō SDE. A standard sufficient condition for existence of a unique (strong) solution to equation (1) is that $f$ and $g$ are globally Lipschitz continuous functions. However, various other (sufficient) conditions have been derived.

Similar to ODEs explicit solutions for SDEs are rarely known, so one has to rely on numerical methods for their simulation. In this context, Maruyama Gishirō [5] extended the Euler scheme for ODEs to SDEs and established its convergence under suitable conditions on $f$ and $g$. For a given grid $0=t_{0}<t_{1}<\cdots<t_{N}=T$ with $N \in \mathbb{N}$, the Euler-Maruyama scheme for SDE (1) reads as

$$
X_{0}=x_{0}
$$

and

$$
X_{k+1}=X_{k}+f\left(X_{k}\right)\left(t_{k+1}-t_{k}\right)+g\left(X_{k}\right)\left(W\left(t_{k+1}\right)-W\left(t_{k}\right)\right), \quad k=0, \ldots, N-1,
$$

where $X_{k}$ is an approximation of $X\left(t_{k}\right)$. Since the early work of Maruyama many contributions followed, which can be roughly divided into two phases. The research of the first phase culminated in the encyclopedic books [6] and [7]. 
Since then, many new topics emerged as, e.g.,

- the numerical analysis of SDEs with an additional jump component (jump diffusions),

- the numerical analysis of SDEs, whose coefficients do not satisfy the standard smoothness assumptions (which quite often arises in real world applications),

- the efficient quadrature of SDEs, e.g., the efficient computation of the expected value $\mathbb{E}(\Phi(X(T)))$ for test functions $\Phi: \mathbb{R} \rightarrow \mathbb{R}$ by means of multi-level Monte Carlo,

- the computation of mean exit times of SDEs,

- the application and simulation of SDEs in chemical kinetics.

These and other topics of this second phase are addressed in this book for the first time outside research articles and monographs. This is a major contribution of the present book. It provides an introduction and overview over these recent developments. After an introduction on random variables and computer simulations (Chap. 1 and 2), Brownian motion, SDEs and further required tools from stochastic analysis are addressed in Chaps. 3-7. The following Chaps. 8-10 study the Euler-Maruyama scheme and its convergence properties, while the remaining chapters deal with the mentioned recent developments (Chaps. 11-16, 20) or more advanced (but classical) topics as higher order schemes or systems of SDEs (Chaps. 17-19).

However, there is another major contribution, which is maybe even more important and makes this book rather unique: It is an introduction to the numerical simulation of stochastic differential equations that is accessible to undergraduate students.

The theory of SDEs is technically very challenging and is typically considered as a topic for advanced graduate students. Additionally, the stochastic nature of SDEs carries over to the numerics of SDEs. Various approximation tasks and concepts (strong approximation, weak approximation, quadrature, approximation of the invariant measure, ... ) exist and many approaches and ideas of the numerics of ODEs fail here. For example, higher order methods are very challenging to construct or even do not exist for particular approximation tasks: For the strong approximation of the Lévy area

$$
\int_{0}^{T} W^{(1)}(t) d W^{(2)}(t)
$$

there are no methods, which rely on evaluations of the two independent Brownian motions $W^{(1)}$ and $W^{(2)}$ at discrete time points and achieve a better convergence order than the simple Euler scheme, see, e.g., [1]. As a further example, the stability analysis of numerical methods has to deal with the stochastic nature of SDEs and uses several stability concepts as mean-square or asymptotic stability, see, e.g., [2].

The authors achieve this accessibility by writing a "non-rigorous book" (using their own words). Instead on technical details they focus on the main ideas and provide many theoretical and computational examples. In particular, each chapter has a key computational topic, which is illustrated by simulations in MATLAB. This hands-on-approach enables the authors to avoid filtrations or other technical details of stochastic analysis and to focus instead on computationally important results. For example, they provide a self-contained proof of the strong and weak convergence 
properties of the Euler-Maruyama scheme (under suitable assumptions on the SDE coefficients $f$ and $g$ and the test functions $\Phi)$, i.e., of

$$
\sup _{k=0, \ldots, N} \mathbb{E}\left|X_{k}-X\left(t_{k}\right)\right| \leq C_{1} \sup _{k=0, \ldots, N-1}\left|t_{k+1}-t_{k}\right|^{1 / 2}
$$

and

$$
\left|\mathbb{E}\left(\Phi\left(X_{N}\right)\right)-\mathbb{E}(\Phi(X(T)))\right| \leq C_{2} \sup _{k=0, \ldots, N-1}\left|t_{k+1}-t_{k}\right|
$$

with $C_{1}, C_{2}>0$.

Sometimes "lack of rigor" is used as a synonym for "carelessness" or "sloppiness". This does not apply to the present book. Many pointers are given to literature, which can be used to fill in the gaps, and careful wording is used throughout the book to remind the reader of its non-rigorous nature. In my opinion, this book is a wonderful and lively introduction into the simulation of SDEs for undergraduate students, but also for researchers, which do not have a background in stochastic analysis. Moreover, even experienced researchers in this field may find many ideas and examples of this book enlightening.

Funding Note Open Access funding enabled and organized by Projekt DEAL.

Open Access This article is licensed under a Creative Commons Attribution 4.0 International License, which permits use, sharing, adaptation, distribution and reproduction in any medium or format, as long as you give appropriate credit to the original author(s) and the source, provide a link to the Creative Commons licence, and indicate if changes were made. The images or other third party material in this article are included in the article's Creative Commons licence, unless indicated otherwise in a credit line to the material. If material is not included in the article's Creative Commons licence and your intended use is not permitted by statutory regulation or exceeds the permitted use, you will need to obtain permission directly from the copyright holder. To view a copy of this licence, visit http://creativecommons.org/licenses/by/ $4.0 \%$.

\section{References}

1. Clark, J.M.C., Cameron, R.J.: The maximum rate of convergence of discrete approximations for stochastic differential equations. In: Stochastic Differential Systems, Proc. IFIP-WG 7/1 Working Conf., Vilnius, 1978. Lecture Notes in Control and Information Sci., vol. 25, pp. 162-171. Springer, Berlin (1980)

2. Higham, D.J.: Mean-square and asymptotic stability of the stochastic theta method. SIAM J. Numer. Anal. 38(3), 753-769 (2000)

3. Ito, K.: Stochastic integral. Proc. Imp. Acad. (Tokyo) 20(8), 519-524 (1944)

4. Ito, K.: On a stochastic integral equation. Proc. Jpn. Acad. 22, 32-35 (1946)

5. Maruyama, G.: Continuous Markov processes and stochastic equations. Rend. Circ. Mat. Palermo 4, 48-90 (1955)

6. Kloeden, P.E., Platen, E.: Numerical Solution of Stochastic Differential Equations. Springer, Berlin (1992)

7. Milstein, G.N., Tretyakov, M.V.: Stochastic Numerics for Mathematical Physics. Springer, Berlin (2004)

Publisher's Note Springer Nature remains neutral with regard to jurisdictional claims in published maps and institutional affiliations. 